\title{
Estudio de la eficiencia instantánea térmica de un campo de colectores cilíndrico parabólico
}

\section{Study of the instantaneous thermal efficiency of a parabolic cylindrical collector field}

\author{
FRANCO-MARTÍNEZ, David †* \& RAMÍREZ-SILVA, Pablo
}

Centro Tecnológico, Facultad de Estudios Superiores Aragón, UNAM

ID $1^{\text {er }}$ Autor: David, Franco-Martínez / ORC ID: 0000-0002-0464-8504, CVU CONACYT ID: 69978

ID $1^{\text {er }}$ Coautor: Pablo, Ramírez-Silva / ORC ID: 0000-0001-5924-1392, CVU CONACYT ID: 897675

DOI: $10.35429 / J O E S .2019 .19 .6 .1 .5$

Recibido 9 de Abril, 2019; Aceptado 30 de Junio, 2019

\section{Resumen}

El objetivo de este artículo es presentar los resultados del estudio de la eficiencia instantánea térmica de un campo de colectores cilíndrico parabólico, que alimentaran de vapor saturado a una microturbina de 5 kw de potencia, tomando en cuenta que la radiación promedio en la Nezahualcoyotl estado de México es de $650 \mathrm{w} / \mathrm{m}^{2}$, el conjunto cuenta con cuatro concentradores, cada concentrador cilíndrico parabólico (CCP) tiene $2 \mathrm{~m}$ de largo y 1,50 m de ancho, con un ángulo de apertura de $90^{\circ}$ y una razón de concentración de 5 , se utilizó aluminio súper pulido con un factor de reflectancia de 0.87 . y la meta es construir un ciclo Rankine simple. Además se ha diseñado y construido un sistema electrónico que tiene la capacidad de seguir de forma autónoma, el sistema de seguimiento funciona sin el uso de sensores fotosensibles para evitar conflictos con el contexto que le rodea y que sea de un costo accesible. De acuerdo a los resultados de las pruebas aplicadas la eficiencia global del campo de colectores solares fue de alrededor del $70 \%$, y el conjunto concentradores generó energía térmica, cercana a $7 \mathrm{~kW}$ térmicos de vapor saturado, requerida por la microturbina de vapor.

Solar, Concentrador parabólico, Seguidor solar

\begin{abstract}
The aim of this article is to present the results of the study of the instantaneous thermal efficiency of a field of parabolic cylindrical collectors, which will feed saturated steam to a micro turbine of $5 \mathrm{kw}$ of power, taking into account that the average radiation in the Nezahualcoyotl state of Mexico is $650 \mathrm{w} / \mathrm{m}^{2}$, the set has four concentrators, each parabolic cylindrical concentrator (CCP) is $2 \mathrm{~m}$ long and $1.50 \mathrm{~m}$ wide, with an opening angle of $90^{\circ}$ and a concentration ratio of 5 , super polished aluminum with a reflectance factor of 0.87 was used to build a simple Rankine cycle. In addition, an electronic system has been designed and built that has the ability to follow autonomously, the tracking system works without the use of photosensitive sensors to avoid conflicts with the surrounding context and that is of an accessible cost. According to the results of the tests applied, the overall efficiency of the solar collector field was around $70 \%$, and the concentrators group generated thermal energy, close to $7 \mathrm{~kW}$ thermal saturated steam, required by the steam micro turbine.
\end{abstract}

Solar, Parabolic concentrator, Solar traker

Citación: FRANCO-MARTÍNEZ, David \& RAMÍREZ-SILVA, Pablo. Estudio de la eficiencia instantánea térmica de un campo de colectores cilíndrico parabólico. Revista de Sistemas Experimentales. 2019. 6-19: 1-5

\footnotetext{
* Correspondencia del Autor (davd_francom@hotmail.com)

$\dagger$ Investigador contribuyendo como primer autor.
} 


\section{Introducción}

Al principio del proyecto se caracterizó un concentrador cilíndrico parabólico (CCP), de 2 $\mathrm{m}$ de largo y 1,20 $\mathrm{m}$ de ancho, con un ángulo de apertura de $90^{\circ}$ y una razón de concentración de 7.6, con tubo de cobre como concentrador de 5 $\mathrm{cm}$ de diámetro, con aluminio súper pulido y una reflexión de 0,8 el cual se utilizó como material reflectante (Figura 1).

Este concentrador generó el vapor de agua como fluido de trabajo para una microturbina de vapor experimental. A partir de estos datos se dimensionó un sistema mas grande para aportar vapor a una microturbina de vapor de $5 \mathrm{~kW}$ de potencia térmica, una ves construido se decidió conocer la eficiencia real del sistema, por lo cual se aplicaron las normas ANSI/ASHRAE, 93-1986 como guía para la evaluación del del campo de colectores, y la Norma NMX-ES-001-NORMEX-2005 para sistemas de calentamiento de agua.

Esto permite tener una metodología de análisis aplicable a cualquier sistema de calentamiento solar, desde el punto de vista termodinámico aplicando un balance de masa y energía.

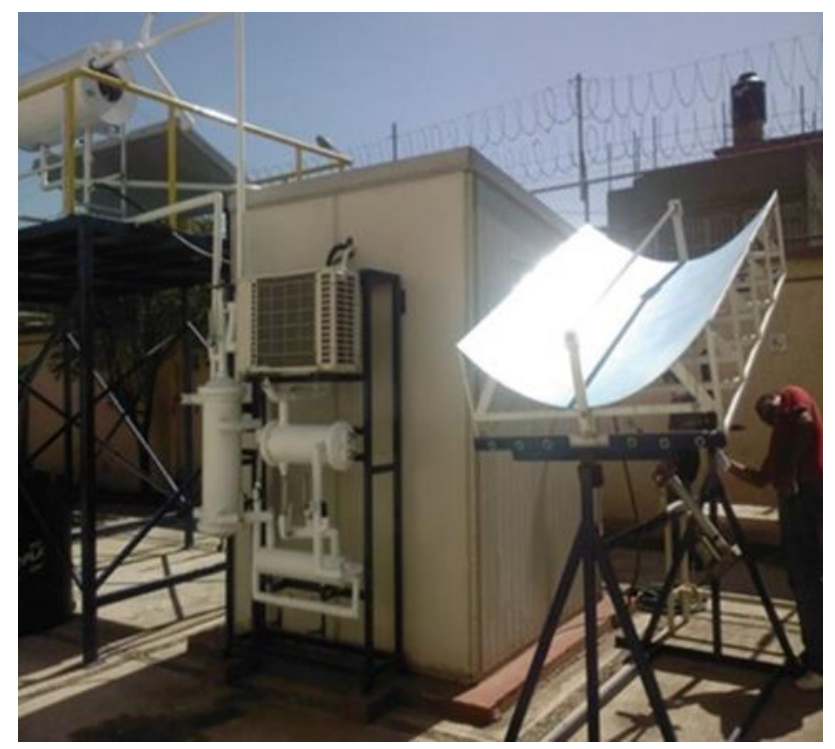

Figura 1 Concentrador parabólico cilíndrico experimental

A continuación, se explican los pasos a seguidos desde el diseño del sistema de colectores solares hasta las pruebas realizadas.

\section{Dimensionamiento del campo de colectores}

A partir de estos resultados se procedió al diseño de la nueva parábola para el campo de colectores, tomando en cuenta la ecuación que la radiación promedio en la Ciudad de Nezahualcoyotl estado de México es de 650 w/m2, promediados con datos de una estación meteorológica en la Facultad de Estudios Superiores Aragón, para 2019.

De acuerdo con los datos generados en el concentrador experimental y tomando en cuenta la eficiencia de la turbina (80\%) para convertir el calor útil del vapor a energía eléctrica, el cálculo del área requerida será expresado con la siguiente ecuación:

$A_{r}=Q_{u} /\left(\eta g_{\text {lobalcolector experimental }} * G b^{*} \eta_{\text {turbina }}\right)$

Donde:

$\mathrm{Qu}$ es el calor útil

$\mathrm{Gb}$ es la radiación solar

Ar es el área bruta del colector

Por lo tanto para poder generar $5 \mathrm{~kW}$ de energía, una eficiencia global del colector esperada del $70 \%$ y una eficiencia estimada de la turbina de $80 \%$, se requerirán un área mínima de $14 \mathrm{~m}^{2}$.

Tomando como base el modelo estudiado se decidió que cada concentrador cilíndrico parabólico $(\mathrm{CCP})$ tendrá $2 \mathrm{~m}$ de largo y $1,50 \mathrm{~m}$ de ancho y un tubo concentrador de cobre para gas de $5 \mathrm{~cm}$ de diámetro y $2 \mathrm{~m}$ de largo, con un ángulo de apertura de $90^{\circ}$, dando una razón de concentración de 9.5, con aluminio súper pulido y una reflexión de 0,8 , por lo que se requerirán cinco concentradores.

\section{Construcción del perfil de la parábola}

Se utilizó un software para obtener las coordenadas de la parábola en función del diámetro y profundidad utilizando la ecuación de la parábola anterior, la cual es:

$(1 / 4) \mathrm{FX}^{2}$

Concluyendo que al utilizarse una placa de aluminio de $0.91 \mathrm{~m}$ de ancho por $3.05 \mathrm{~m}$ y un espesor de $0.98 \mathrm{~mm}$, debíamos obtener 4 bastidores para el soporte de cada colector por placa, se ajustaron las dimensiones de la parábola de acuerdo con los siguientes datos:

FRANCO-MARTÍNEZ, David \& RAMÍREZ-SILVA, Pablo. Estudio de la eficiencia instantánea térmica de un campo de colectores cilíndrico parabólico. Revista de Sistemas Experimentales. 2019 


\section{- Diámetro: $150 \mathrm{~cm}$ \\ - $\quad$ Profundidad: $40 \mathrm{~cm}$}

Obteniéndose una distancia focal: 35.15 $\mathrm{cm}$, para no desperdiciar material y obtener un área de $3 \mathrm{~m}^{2}$ por parábola.

Dicha ecuación con coordenadas sirvieron para dibujar la curva en AutoCAD, mediante la función spline. Una vez obtenida la curva, se dibujó el esbozo de los soportes del panel, así como se muestra en la imagen (Figura 2 y 3$)$.

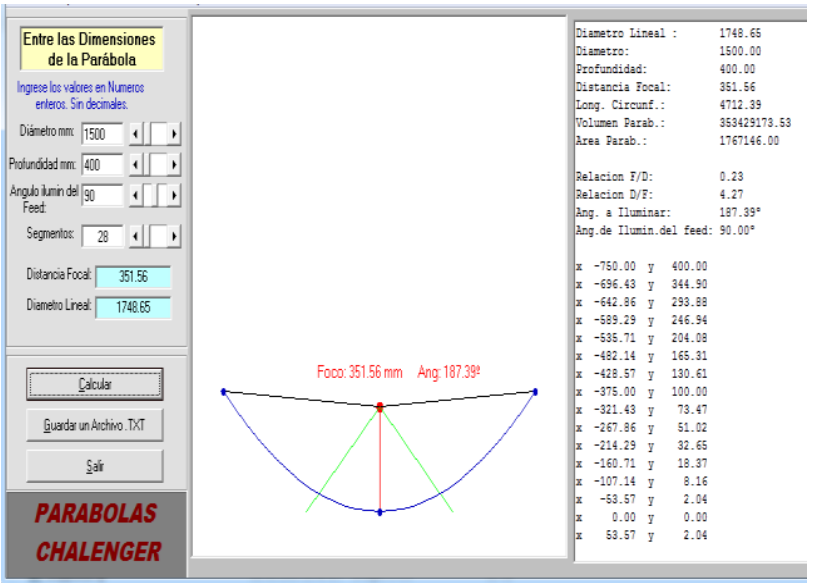

Figura 2 Parabola dibujada con Auto CAD

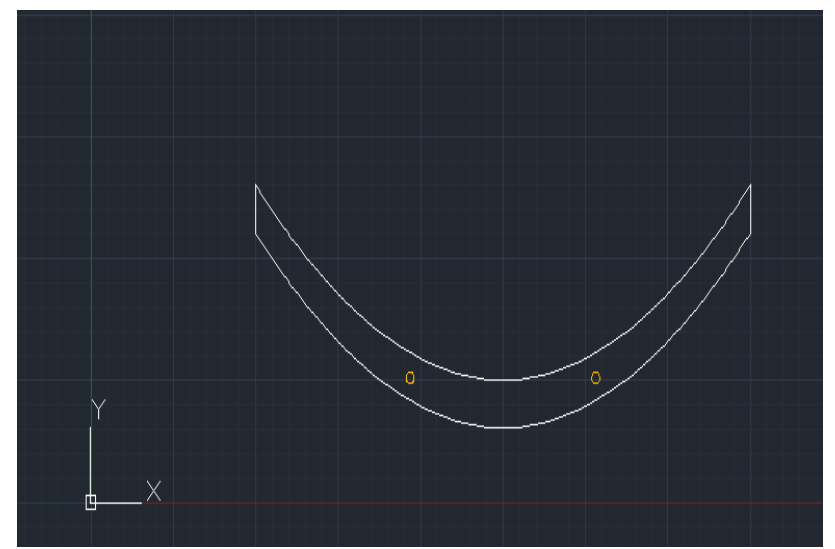

Figura 3 Perfil de la parábola

Se realizaron pruebas de funcionamiento acoplado a un sistema de almacenamiento de agua (termo tanque) junto con el seguidor solar y probar la eficiencia del sistema total de calentamiento de agua Ver figura 4.

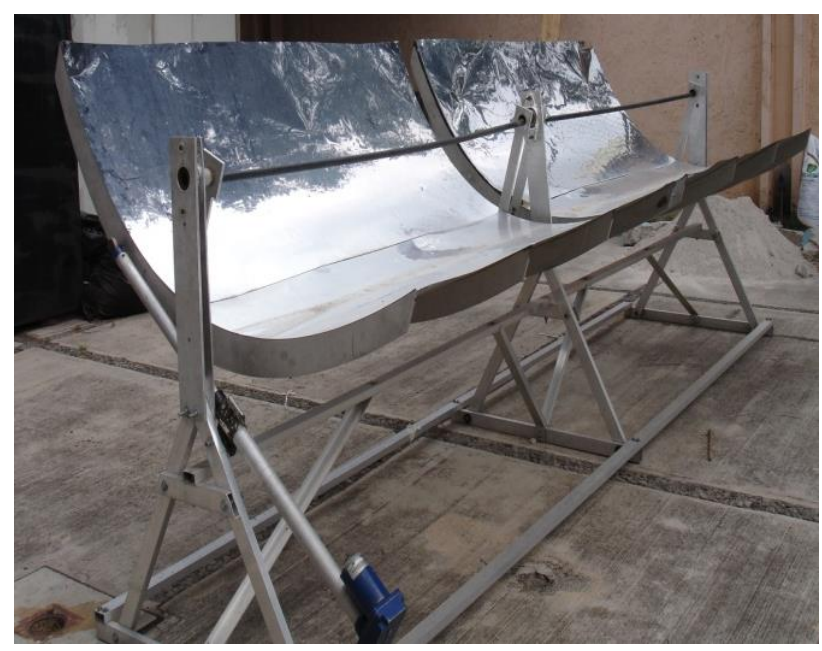

Figura 4 Prototipo final acoplado en la estructura diseñada

La norma ANSI/ASHRAE, 93-1986 se utilizó como guía para la evaluación del CCP. Así también, se utilizaron las pruebas experimentales del calentamiento de agua, para la aplicación de la Norma NMX-ES-001NORMEX-2005. A continuación, se describen en forma específica los pasos a seguir.

\section{Esquema general del equipo}

En la figura 5 se observa el esquema del dispositivo experimental que se implementó para la evaluación del $\mathrm{CP}$, requerido para poder realizar la colocación de equipos de medición y poder obtener los datos para calcular la eficiencia instantánea del sistema.

Los principales componentes son:

1. Tanque de almacenamiento de agua. Para la suministrar agua al sistema y recircularla

2. Bomba de agua Marca SIEMENS Motor eléctrico monofásico de $1 / 4$ HP Monofásica Centrifuga Conexiones Succión 1 1/4", Descarga 1" F.S 1.30 PRM. 3475 Voltaje $127 \mathrm{v}-220 \mathrm{v} \mathrm{Hz} 60$ Corriente Nominal A 12,5/6,0 Corriente FS (A) 14,0/8,0; para comprimir el fluido de trabajo, mantener el ciclo de trabajo de prueba.

3. Válvulas de aguja y de compuerta para desviar el flujo.

4. Medidores de presión o traductores de presión.

5. Termopares de temperatura. 
6. Sistema de adquisición de datos LabVIEW, con un complemento de software que ayuda a realizar muchas operaciones de base de datos para conectarse fácilmente a bases de datos en una hoja de Excel para calcular la eficiencia instantánea del sistema de concentración.

7. Anemómetro, equipo medidor de la velocidad de aire.

8. Rotámetro Flujómetro FLOCAT en Polysulphone. Para AGUA, Rango: 4 18 GPM se determina el flujo de agua durante las pruebas

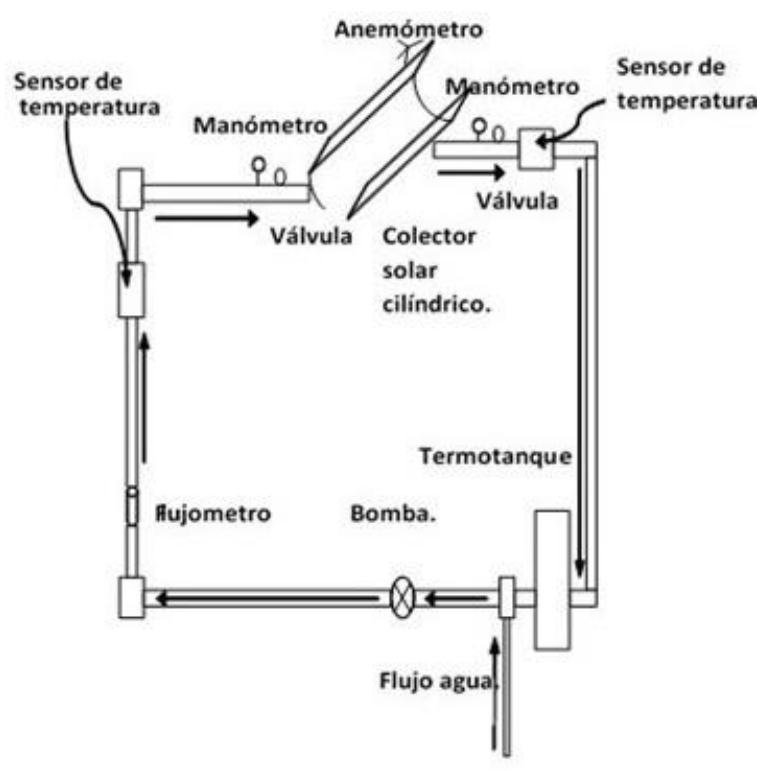

Figura 5. Esquema del equipo utilizado.

\section{Resultados}

Las pruebas experimentales realizadas al campo de concentradores solar consistieron en determinar los servicios globales ofrecidos para el calentamiento de agua, que incluyen la determinación de la eficiencia instantánea de concentrador con la radiación normal a la superficie colectora, la medición de la capacidad calorífica efectiva del concentrador y la determinación de la constante de tiempo. La prueba se realizó el día 7 de mayo, en la figura 6 se puede observar la radiación solar:

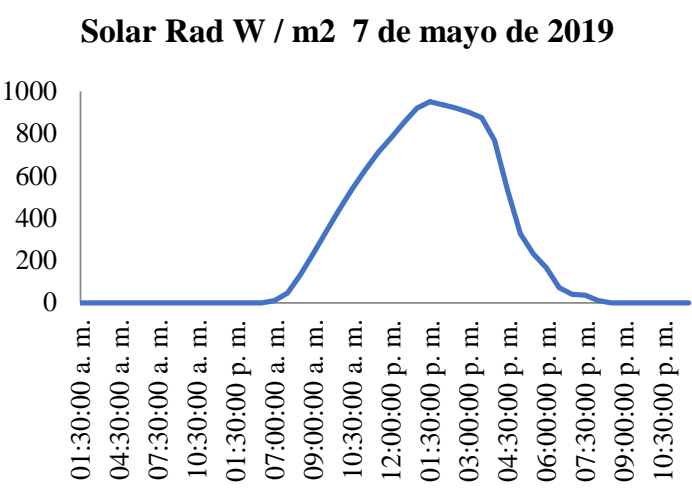

Gráfico 1 Irradiación solar del 7 de mayo en la FES Aragón

Conforme el fluido fluye a través del receptor, este va ganando calor provocando que el flujo másico no sea uniforme, a consecuencia de esto, el factor FR es variable y así mismo el rendimiento térmico del sistema. El factor FR es una función del valor del flujo, en los calentadores de agua y los cambios en el flujo son una diferencia significativa. En el rendimiento del colector. Además las pérdidas de calor en los tubos, son significativas, debido a que no están aislados y las longitudes son grandes.

El rendimiento térmico del colector solar se determina mediante la obtención de valores de eficiencia instantánea de una combinación de valores de radiación incidente, temperatura ambiente, y la temperatura del agua de entrada. Esto requiere medir experimentalmente la radiación solar incidente sobre el colector solar, así como la tasa de incremento de la energía calorífica en el agua mientras pasa a través del colector solar, todo bajo condiciones de estado estacionario o cuasi-estacionario.

El método experimental de acuerdo a la norma NMX-ES-001-NORMEX-2005 para realizar las mediciones, debe ser en estado estable o casi-estacionario, siendo los parámetros a registrar la temperatura de entrada y salida del fluido de trabajo y determinar el calor útil ganado $Q u$. Así también, se determina la eficiencia instantánea del colector.

$\eta=Q u / A c G b$

Donde:

Qu es el calor util

$\mathrm{Gb}$ es la radiación solar

Ac es el área bruta del colector. 
Donde la Ec. (3), es para evaluar el calor útil, que depende de la temperatura de entrada y salida del fluido y del flujo másico que circula a través del colector.

$Q u=m C p($ Tsalida-Tentrada $)$

Por lo que la eficiencia se puede calcular como:

$\eta=m C p($ Tsalida-Tentrada $) / A c G b$

Al graficar los resultados obtenemos los valores de la figura 7 .

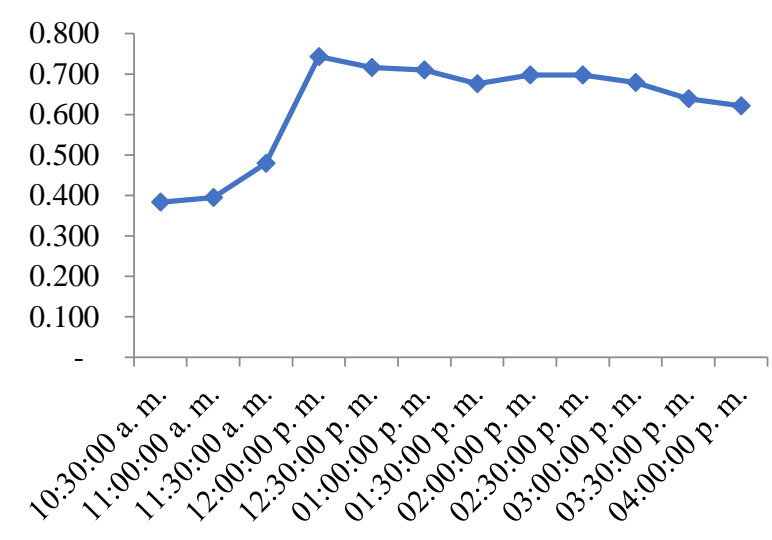

Gráfico 2 Eficiencia experimental medida para un campo CCP. Prueba del 7 de mayo de 2019.

\section{Conclusiones}

Cabe señalar que las eficiencias reportadas para el campo de colectores se vieron afectada por las pérdidas por convección ya que el concentrador no se encuentra encapsulado en un tubo de vidrio, lo cual disminuiría estas pérdidas. Las eficiencias térmicas del sistema de concentración estuvieron en promedio del $70 \%$. El objetivo del proyecto fue el de generar una metodología de análisis de sistemas de concentración solar y generar material para nuevas experimentaciones en esta energía renovable, ya que en la literatura no existe una forma práctica de evaluación de sistemas de concentración.

Se manejó un flujo relativamente bajo (0.575 litros por minuto) y lo que que se deben realizar pruebas para flujos mayores, las cuales no se pudieron realizar por falta de tiempo, y es obvio que la eficiencia bajara. Se recomienda aumentar a 3 colectores más para alcanzar condiciones de vapor mejores

\section{Referencias}

Birnbaum J., Feldhoff J., Fitchner M., Hirsch T., Jöcker M., Pitz-Paal R., and Zimmermann G., "Steam temperature stability stability in a direct steam generation solar power plant", Solar Energy Vol 85, 2011, 660 - 668.

Duffie A. J., Beckman A. W., Solar Engineering of Thermal Processes, 3rd edition, John Wiley and Sons, Inc., Wisconsin, 2006.

Eck M., Zarza E., "Saturated steam process with direct steam generating parabolic troughs", Solar Energy, Vol. 80, 2006, 1424 - 1433.

Enciso Contreras E., Desarrollo de un software para el diseño de concentradores solares de canal parabólico, tesis de maestría, SEPI - ESIME Zacatenco, Instituto Politécnico Nacional, Ciudad de México, 2012.

García-Valladares O., Velázquez N., "Numerical simulation of parabolic trough solar collector: Improvement using counter flow concentric circular heat exchangers", International Journal of Heat and Mass Transfer, Vol. 52, 2008, 597 - 609.

Hottel H. C., "A simple Model for estimating the transmittance of direct solar radiation through clear atmospheres", Solar Energy, Vol. 18, 1976, $129-134$.

Incropera F. P., DeWitt D.P., Fundamentals of Heat and Mass Transfer, 6th edition, John Wiley and Sons, New York, 2007.

Kalogirou S., Solar Engineering Processes and Systems, 1st. edition, Elsevier Inc., New York, 2009.

Kalogirou S., Lloyd S., Ward J., "Modelling, optimization and perform 